\title{
Comparing pediatric intentional injury surveillance data with data from publicly available sources: consequences for a public health response to violence
}

\author{
David A Stone, Sigmund J Kharasch, Catherine Perron, Kim Wilson, Beth Jacklin, \\ Robert D Sege
}

Department of Pediatrics, Pediatric and Adolescent Health Research Center, Floating Hospital for Children at New England Medical Center, Tufts University School of Medicine

D A Stone R D Sege

Pediatric Emergency Medicine, Boston Medical Center, Department of Pediatrics, Boston University School of Medicine

S J Kharasch

Department of Pediatrics, Division of Emergency Medicine, Children's Hospital, Harvard University

C Perron

Children's Hospital, Martha Elliot Health Center

$\mathrm{K}$ Wilson

Pediatric and Adolescent Health

Research Center, Floating Hospital for Children at New England Medical Center

B Jacklin

Correspondence to: Dr Robert D Sege, Pediatric and Adolescent Health Research Center, New England Medical Center, Box 531, 750 Washington Street, Boston, MA 02111, USA (e-mail:

robert.sege@es.nemc.org).
Abstract

Objective-A hospital based intentional injury surveillance system for youth (aged 3-18) was compared with other publicly available sources of information on youth violence. The comparison addressed whether locally conducted surveillance provides data that are sufficiently more complete, detailed, and timely that clinicians and public health practitioners interested in youth violence prevention would find surveillance worth conducting. Setting-The Boston Emergency Department Surveillance (BEDS) project was conducted at Boston Medical Center and the Children's Hospital, Boston.

Method-MEDLINE and other databases were searched for data sources that report separate data for youth and data on intentional injury. Sources that met these criteria (one national and three local) were then compared with BEDS data. Comparisons were made in the following categories: age, gender, victim-offender relationship, injury circumstance, geographic location, weapon rates, and violent injury rates.

Results-Of 14 sources dealing with violence, only four met inclusion criteria. Each source provided useful breakdowns for age and gender; however, only the BEDS data were able to demonstrate that $32.6 \%$ of intentional injuries occurred among youth aged 12 and under. Comparison data sources provided less detail regarding the victim-offender relationship, injury circumstance, and weapon use. Comparison of violent injury rates showed the difficulties for practitioners estimating intentional injury from sources based on arrest data, crime victim data, or weapon related injury.

Conclusions-Comparison suggests that surveillance is more complete, detailed, and timely than publicly available sources of data. Clinicians and public health practitioners should consider developing similar systems.
(Injury Prevention 1999;5:136-141)

Keywords: violence; surveillance; youth; emergency department

Surveillance, begun in the 1950 s and 1960 s to control epidemics of smallpox, cholera, and other infectious diseases, has since proved itself valuable in efforts to reduce injury. ${ }^{1}$ More specifically, hospital and emergency department based surveillance efforts have described and tracked the incidence of injury, identified specific risk factors, and supported prevention interventions in local settings. ${ }^{2-7}$ This paper attempts to show the relative value of undertaking emergency department based surveillance for intentional injury among youth in an urban setting compared with other data currently available for use by clinicians and public health practitioners.

Current public health efforts aimed at the prevention of violence related injury among youth use data from a variety of sources collected in different ways for a number of different purposes (for example, police data, crime surveys, hospital discharges). However, none of these sources specifically measures the incidence of violence related injury to children and adolescents. This lack forces clinicians and public health practitioners working on youth violence to rely on estimates of injury based on such proxies as homicides and reported violent crimes.

This gap has been noted by the Centers for Disease Control and Prevention (CDC). In Violence in America, Rosenberg states that "data needs are much greater for nonfatal assault violence than for homicide... there is an urgent need at both the national and local levels for better information on injuries resulting from nonfatal assaults". ${ }^{8}$ Youth homicide surveillance has enabled risk group and risk factor identification and the implementation and evaluation of programs designed to reduce youth homicide. Guyer et al have reported similar success in the area of unintentional injury. ${ }^{9}$ We believe that intentional injury surveillance could permit identification of risk 
group and risk factor characteristics as has been accomplished for youth homicide and unintentional injury.

It can be argued that data sufficient for a public health response to intentional injury can be obtained by synthesizing the information provided by data that are currently publicly available and that surveillance is otiose. This paper addresses that argument by comparing the information gained from emergency department surveillance in one city (Boston) with other, publicly available data. At issue in the comparison are:

- The presence or absence of data collected on variables represented in the current youth violence literature as being associated with risk for intentional injury.

- How well each data source covers the range of types and levels of youth injury.

- The level of detail available within each of the reported variables for each data source.

- The timeliness of the data reported by each source.

We report the results from the first year of the Boston Emergency Department Surveillance (BEDS) project conducted in the emergency departments of two Boston hospitals: Boston City Hospital (now Boston Medical Center) and the Children's Hospital. These data are then compared with existing publicly available data on violence related injury among youth.

\section{Methods}

IDENTIFYING AVAILABLE DATA ON YOUTH

VIOLENCE

We define "publicly available" as data that were easily obtainable either in published form or in response to a simple telephone request. The purpose of our method was to mimic the circumstances of a clinician, researcher, or public health provider seeking data for a funding proposal or for planning a local violence prevention intervention. We employed two search methods: (1) MEDLINE, using combinations of the following keywords: injury, rate, scope, pediatric, assault, violence, intentional, 1990-96 and (2) readily available secondary sources (for example, Violence in America: $A$ Public Health Approach ${ }^{8}$ and Youth Violence: Locating and Using Data ${ }^{10}$ ).

DATA REVIEW AND SELECTION PROCESS

We selected data sources for the comparison based on the following criteria: (1) the sources include separate statistics for children and youth under age 19 and (2) the sources differentiate intentional and unintentional injury. We solicited data from both national and local sources and, when necessary, contacted data sources directly.

For each source, we sought to obtain the following information: victim demographic characteristics, circumstances of injury, victimoffender relationship, time and place of the incident, and weapon use. Each of these variables is regarded in the literature as important for the identification of risk groups and risk factors associated with violent injury. ${ }^{11-17} \mathrm{In}$ addition, we sought data on the type of injury sustained and procedures performed.

\section{BOSTON EMERGENCY DEPARTMENT}

SURVEILLANCE

The purpose of the BEDS project is to establish an ongoing citywide surveillance system to track the nature and scope of violent injury among youth and to monitor changes in violent injury over time. Unlike other measures of community violence, BEDS focuses specifically on intentional injuries of sufficient severity to warrant emergent medical treatment. We identified patients either prospectively (at triage) or retrospectively by ongoing, daily emergency department record review. We defined violence related injuries as those resulting from a situation of conflict involving two or more persons with intent to harm. BEDS focus is on "street violence". For this reason, BEDS excludes injuries resulting from child abuse (by International Classification of Diseases, ninth revision (ICD-9) code E967), those in which the other person involved in the incident leading to injury was identified as parent, those resulting from attempted suicide, and those where intent is unclear.

The BEDS data used in this paper cover data collected from 1 April 1995 to 31 March 1996. The total number of cases included is 677 . Retrospective chart reviews for this period indicate that BEDS captures over $95 \%$ of intentional injury cases at each hospital.

Forms previously used in our own studies ${ }^{18} 19$ and those conducted by the Massachusetts Weapons Related Injury Surveillance System (WRISS) $^{20}$ served as models for the forms developed for this project. The forms record data routinely obtained in the normal course of emergency department care in seven domains: (1) patient demographics (age, sex); (2) injury circumstance (argument, crime related, bystander, etc); (3) victim-offender relationship; (4) time of incident and victim place of residence; (5) assessment of drug use by the victim; (6) medical description of injury and procedures (diagnosis, ICD-9 code, ICD-9 E code); and (7) whether a weapon was involved in the incident. The forms were pilot tested before use.

Study staff entered results into a Microsoft Access database and verified these using the original form. We conducted data analysis using Epi-Info and SAS. ${ }^{21}$

Rates were calculated using 1990 US Census data for the Boston population aged $3-18$ as the denominator $(n=113033)^{22}$ and injuries involving patients living in Boston $(n=560)$ as the numerator, noting that Boston City Hospital and the Children's Hospital together treat approximately $82.9 \%$ of intentional injuries among youth treated in Boston hospital emergency departments (based on data collected from the four major hospitals that treat children). Boston City Hospital treated 383 patients $(56.6 \%)$ and the Children's Hospital treated 294 patients $(43.4 \%)$.

The human studies committees at $\mathrm{New}$ England Medical Center, Boston City Hospi- 
Table 1 Age distribution of violence related injuries for different reporting sources. Data from the Boston Emergency Department Surveillance for the age categories of each source are given for comparison

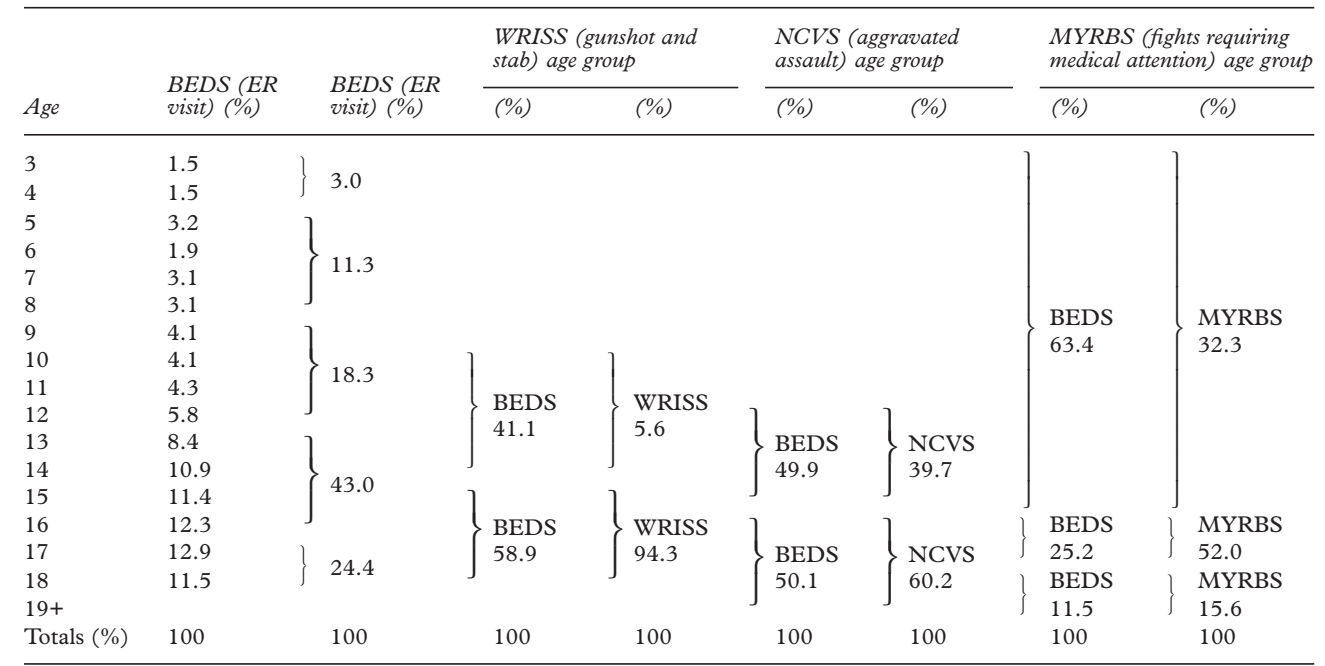

BEDS = Boston Emergency Department Survey.

WRISS $=$ Weapon Related Injury Surveillance System NCVS = National Crime Victimization Survey.

MYRBS = Massachusetts Youth Risk Behavior Survey.

tal, and the Children's Hospital, Boston, approved this study.

\section{Results}

COMPARISON OF DATA SOURCES

We identified one national data source and three state and local data sources that met our general criteria for usefulness and public availability. The national source, the National Crime Victimization Survey (NCVS) ${ }^{23}$ annually collects data through in-person and phone interviews of a nationally representative sample of 120000 household occupants over the age of 12 .

In addition, we identified several local data sources for comparison to the BEDS data. WRISS is a CDC funded statewide surveillance program that identifies all individuals treated as the result of gunshot or stab wounds. We used 1995 WRISS data on violence related injury among youth (aged 1-18) in Boston $(n=125)$. The Massachusetts Youth Risk Behavior Survey (MYRBS) is a CDC funded biennial survey of youth attending high school and we used the city of Boston results for 1995 $(n=4089){ }^{24}$ Finally, we included crime data from the Boston Police Department from their 1993 report (Boston Police Department, Crime Data Summary, June 1994) $(\mathrm{n}=1352)$.

The following sections compare the findings from the BEDS project with each of these sources using the four criteria described above. The purpose of the comparisons is to show how different the local picture of intentional injury is when hospital surveillance is used rather than other publicly available data focused more generally on youth violence.

AGE

Understanding the distribution of injury by age may be crucial in identifying the onset of serious involvement with violence and its progression, as well as in the development and targeting of intervention strategies. Only two of the publicly available data sources provide categorical breakdowns of injury data by age. BEDS provides data for five age categories, beginning with youth age 3. Altogether $32.6 \%$ of those treated in emergency departments were aged 12 and younger and this figure may be an overestimate because older youths are sometimes seen in adult emergency departments (see table 1).

WRISS reveals a different age distribution; $94 \%$ of youth injured with a weapon were between the ages of 15-18. Among BEDS cases, only $17.1 \%$ would meet WRISS criteria, and of those, only $55.2 \%$ were aged 15-18. (Note that in comparisons between the WRISS and BEDS data sets, the term weapon related employs the WRISS definition, which means gunshot or stab wounds only.) Finally, the NCVS reports data on aggravated assaults (assaults with a dangerous weapon) but like WRISS, only in two age categories for youth 12 or older.

The MYRBS tracks injury from a physical fight requiring the attention of a doctor or nurse. The MYRBS reports such injury (at least once) for youth 15 or younger $(4.6 \%)$, youth 16 or $17(4.3 \%)$, and youth 18 or older (3.9\%). The Boston Police Department aggregates reports on aggravated assault for youth under 18 , so no age specific data are available.

\section{GENDER}

As more young girls become involved in violence (heterosexual dating violence, girl on girl violence, and gang activity), it is important to track violence by gender. BEDS data indicate that $35.5 \%$ of injured youth were female. This percentage is fairly consistent across all ages. For youth with weapon related injuries in the BEDS data set, $25.3 \%$ were female.

By contrast, WRISS data indicate that of those injured by weapons, only $9.8 \%$ were female. MYRBS data show that while $49.3 \%$ of 
Table 2 Comparison of rates for injuries and other violent encounters

\begin{tabular}{|c|c|c|}
\hline Data source & $\begin{array}{l}\text { Rate/10 000 youth } \\
(95 \% \text { CI })\end{array}$ & Type of outcome \\
\hline BEDS & $72(67$ to 77$)$ & Injuries treated in emergency department \\
\hline MYRBS survey & $570(499$ to 641$)$ & Physical fights \\
\hline Boston Police Department survey* & $700(521$ to 879$)$ & Assault victims \\
\hline Hausman’s survey* & $260(105$ to 415$)$ & Fights requiring medical attention \\
\hline Boston Police Department crime reports (all reported crimes) & 78 & Aggravated assault \\
\hline WRISS emergency department data (all reported injuries) & 11 & Gunshot and stab wounds \\
\hline NCVSt & $776(761$ to 791$)$ & Simple assault $\ddagger$ aged $12-15$ \\
\hline NCVS & 711 (696 to 726$)$ & Simple assault aged $16-19$ \\
\hline NCVS & $222(214$ to 230$)$ & Aggravated assault $₫$ aged $12-15$ \\
\hline NCVS & 337 (327 to 347$)$ & Aggravated assault aged 16-19 \\
\hline
\end{tabular}

*Table 2 includes data from two additional sources: (1) a Boston Police Department survey of high school students ( $\mathrm{n}=781)$ and (2) a random digit phone survey conducted by Hausman et al. ${ }^{11}(\mathrm{n}=404)$. We chose to include these data because they represent a type of local data often used by policymakers and providers to estimate levels of injury in specific communities.

tConfidence interval (CI) based on the total NCVS sample of 120000 individuals.

$¥$ Simple assault (or assault) is assault without a weapon.

§Aggravated assault is assault involving a weapon.

male students reported having a physical fight in the past year, $30.7 \%$ of female students so reported. A similar disparity is found regarding the need for medical attention (males $7.9 \%$, females 3.5\%). The Boston Police Department reported that crimes of violence for youth under 18 involved $53 \%$ males, $47 \%$ females as victims. The NCVS reports that among aggravated assault victims, those $12-15$ years old were more likely to be male $(63.8 \%)$ as were those aged 16-19 (69.1\%). Injuries among BEDS cases for these ages groups were $65.5 \%$ males (aged 12-15) and 63.4\% males (aged 16-18).

VICTIM-OFFENDER RELATIONSHIP

Data on the victim-offender relationship, especially in adult homicide, has helped reduce concerns about stranger violence and has increased awareness of dating violence and spouse abuse. Victim-offender relationship information may isolate risk factors and aid in developing effective interventions.

According to BEDS data, the relationship between the patient and the other person involved in the incident was recorded for $67 \%$ of visits. BEDS collects victim-offender data in nine categories. Among youth reporting a relationship, friends or schoolmates combined to account for roughly $40 \%$ of cases, while "strangers" accounted for only $6.4 \%$ of cases. For youth injured by weapons, only $62.9 \%$ reported being injured by friends or schoolmates while strangers accounted for $9.7 \%$ of cases.

Data from the MYRBS is similar. For those reporting on their last physical fight the results are: someone they knew (males $43.2 \%$, females $34.0 \%$ ); stranger (males $21.2 \%$, females $6.7 \%$ ); or family member (males $7.3 \%$, females $35.5 \%$ ). For both WRISS and NCVS, strangers played a much larger part: WRISS aged 15-18, 31.9\%; NCVS aged 12-15, 34.5\%; and NCVS aged 16-19, 46.8\%. The Boston Police Department does not report these data for youth.

INJURY CIRCUMSTANCE

Previous reliance on police data has resulted in a view of violence as mostly crime and gang related. Detailed data on the circumstances of injury should permit a sharper focus on areas of risk and avenues for prevention.
BEDS obtained data on injury circumstance for $95.3 \%$ of cases. Injuries resulted most often from arguments (36.6\%) followed by those that were unprovoked $(9.9 \%)$, gang related $(4.5 \%)$, or crime related $(4.0 \%)$. For youth with weapon related injuries (excluding blunt instruments), the percentage of gang related incidents nearly doubles $(9.6 \%)$ while the proportions of other injury circumstances remain roughly the same.

For youth 15-18, WRISS reported that $21 \%$ of weapon related injuries resulted from arguments, $8 \%$ other crimes, $9 \%$ other, and $62 \%$ missing. The Boston Police Department does not report these data for youth and NCVS provides no information on circumstance or victim-offender relationship.

\section{WEAPON USE}

BEDS data indicate that weapons contributed to a minority of injuries treated in the emergency department: gunshot $4.3 \%(n=29)$, knife/sharp instrument $13.0 \% \quad(n=88)$, blunt instrument $13.9 \%(n=94)$, and $49.0 \%(n=332)$ unarmed fights. WRISS data, which tracks only weapon related injury identified 49 gunshot wounds $(39.5 \%)$ and 75 sharp instrument wounds $(60.5 \%)$ among youth treated at Boston hospitals. Neither the Boston Police Department nor the NCVS report weapon specific data.

\section{VIOLENCE RELATED INJURY RATES}

Another important tool for a public health approach is an accurate and timely measure of incidence. However, the lack of available data specific to injury has required researchers, providers, and policy makers to rely on proxies for injury from related circumstances like fights or reported assaults. Table 2 highlights the variation across such proxy measures as well as the difficulty of inferring the rate of injuries from such disparate sources.

\section{Discussion}

Public health efforts to reduce levels of violent injury among youth require thorough, detailed, and timely data about the scope and nature of violent injury in communities targeted for intervention, and a method for monitoring the success or failure of these efforts. This paper compares the publicly available data with that 
compiled from our surveillance project. In making these comparisons, we recognize that each of these sources collects data specific to its own purposes. We are thus making no claim that the BEDS data are generally superior to these other sources. Furthermore, this paper does not address the accuracy of the BEDS data, which must await further study.

PRESENCE OR ABSENCE OF INTENTIONAL INJURY DATA

The scope and nature of intentional injury among youth provided by the publicly available data sources is fragmentary and, in many cases, differs in important respects from the BEDS data. For each of the criteria used in our comparisons, completeness, detail, and timeliness, the publicly available data sources exhibited limitations that reduce their utility in assisting providers and policy makers to develop, implement, and monitor youth violence prevention interventions.

Our comparison highlights the paucity of complete, detailed, and reliable data available on pediatric injury at both the national and local levels. Only four of 14 violence related data sources met the prima facie requirements of including separate data on youth and discriminating between intentional and unintentional injury.

COMPLETENESS

Completeness refers to the range of type and severity of intentional injury covered by a given data source. The publicly available data sources tend to focus on a fairly narrow range of either the type or severity of injury (that is, weapon related; crime related, homicides, physical fights, adolescents only, etc) none of which, as we have shown, reveals the full scope and nature of violent injury among youth. BEDS data, on the other hand, cover all injuries for which emergency medical attention was sought.

DETAIL

Detail refers to the number of data elements within each of the variables for which data were obtained. We chose variables for comparison that have been associated with intentional injury in previous studies. With respect to age, only BEDS and WRISS cover the full range of ages up to 18. Although WRISS includes youth under age 12, young children are less likely to be injured by an armed assailant and thus less likely to be included. Thus, because younger children are less likely to be injured with a weapon, only BEDS revealed that one third $(32.6 \%)$ of injuries were in children aged 12 and under.

For those data sources that reported information about the nature of the violent incident, injury circumstance (BEDS, WRISS), and the victim-offender relationship (BEDS, WRISS, MYRBS, NCVS), each provided a level of detail sufficient to identify the frequency of common risk characteristics. However, again because only $17.1 \%$ BEDS cases were weapon related, the data provided by WRISS for injury circumstance and victim-offender relationship only account for a small percentage of those injured.

The lack of detail available from other data sources with respect to both the victim (age, gender) and the incident (victim-offender relationship, injury circumstance, injury severity, and geography) make univariate or multivariate analyses of specific risk factors impossible in most cases. Additionally, unlike the other sources, BEDS provided detailed information on the type of injury (level of service, E code, diagnosis, special procedures, patient's disposition, and follow up recommendations) and medical services received. Within the emergency department setting, these data are useful for tracking trends in injury types. They have also been used as a catalyst for continuing staff education, as a method for monitoring appropriate documentation and treatment, and as a method for assessing the impact of youth violence on emergency department services.

TIMELINESS

All of the data sources employed in this comparison provide data within two years from the time it was collected. However, BEDS permits access to current data and the ability to parse the data into monthly or weekly segments to identify temporal trends (both secular and cyclical).

COSTS AND BENEFITS

Although we believe that the data provided are useful, there are substantial costs associated with the implementation of citywide surveillance of violence related injuries. Specifically, these include: (1) data collection; (2) training to improve identification of intentionality; and (3) data entry and cleaning.

The benefits of conducting intentional injury surveillance include the ability to supply data to public health and public safety planners that permits them to target interventions to specific age groups, genders, geographic locations, as well as toward youth who share other identified risk characteristics. For example, BEDS data on re-injury, showing a sixfold increase in the risk for re-injury, has been used to develop a program targeting injured youth for prevention of re-injury.

\section{Implications for prevention}

In sum, the BEDS data provide a much broader and more detailed picture of the scope and nature of violence in the community than is obtainable through the use of such proxies as homicide or crime data. Patients are younger, more likely to know who hurt them, and less likely to be the victims of armed assault than is suggested by the available data sources. They are also at high risk of re-injury. Such findings, as well as the ability to monitor changes in these findings over time, have already been used to inform the development of specific prevention interventions as well as helping to monitor their success.

1 Graitcer PL. The development of state and local injury surveillance systems. F Safety Res 1987;18:191-8. 
2 Zwi K, Zwi A, Smettanikov E, et al. Patterns of injury in children and adolescents presenting to a South African

3 Kraus J, Hooten E, Brown K, et al. Child pedestrian and bicyclist injuries: results of community surveillance and a case-control study. Inj Prev 1996;2:212-18.

4 Weiss H. Limitations of child injury data from the CPCS's National Electronic Injury Surveillance System: the case of baby walker related data. Inj Prev 1996;2:61-6.

5 Agran P, Winn D, Anderson C. Surveillance of pediatric injury hospitalizations in Southern California. Inj Prev 1995;1:234-7.

6 Lyons R, Lo S, Heaven M, et al. Injury surveillance in children-usefulness of a centralised database of accident and emergency attendances. Inj Prev 1995;1:173-6.

7 Barancik J, Kramer C. Northeastern Ohio trauma study: overview and issues. Public Health Rep 1985;100:563-4.

8 Rosenberg ML, Fenley MA, eds. Violence in America: a public health approach. New York: Oxford University Press, 1991.

9 Guyer B, Lescohier I, Gallagher SS, et al. Intentional injuries among children and adolescents in Massachusetts. injuries among children and adol

10 Posner M. Youth violence: locating and using the data. Newton, MA: Children's Safety Network/Education Development Center, 1996

11 Hausman AJ, Spivak H, Prothrow-Stith D. Adolescents' knowledge and attitudes about and experience with violence. F Adolesc Health 1994;15:400-6.

12 Li G, Baker SP, DiScala C, et al. Factors associated with the intent of firearm-related injuries in pediatric trauma patients. Arch Pediatr Adolesc Med 1996;150:1160-5.

13 Saner H, Ellickson P. Concurrent risk factors for adolescent violence. $\mathcal{F}$ Adolesc Health 1996;19:94-103.

14 DuRant RH, Kahn J, Beckford PH, et al. The association of weapon carrying and fighting on school property and other health risk and problem behaviors among high school students. Arch Pediatr Adolesc Med 1997;151:360-6.

15 Finkelhor D, Asdigian NL. Risk factors for youth victimization: beyond a lifestyles/routine activities theory approach. Violence Vict 1996;11:3-19.

16 Garmezy N. Children in poverty: resilience despite risk. Psychiatry 1993;56:127-35.

17 Hartzog TH, Timerding BL, Alson RL. Pediatric trauma: enabling factors, social situations, and outcome. Acad Emerg Med 1996;3:213-20.

18 Sege R, Stigol LC, Perry C, et al. Intentional injury surveillance in a primary care pediatric setting. Arch Pediatr Adolesc Med 1996;150:277-83.

19 Kharasch S, Perron C, Wilson K, et al. Kids hurting kids: injuries due to peer violence (abstract). Washington, DC: Ambulatory Pediatrics Association, 1-5 April 1996.

20 Ozonoff V, Barber C, Hume B, et al. Emergency department surveillance for weapon-related injuries-Massachusetts, November 1993-April, 1994. Morb Mortal Wkly Rep 1995; 44:160-9.

21 SAS. SAS version 6.12. Cary, NC: SAS Institute, 1996.

22 Bureau of the Census. 1990 census of population and housing: summary social, economic, and housing characteristics for Massachusetts. Washington, DC: United States Department of Commerce, 1992

23 Bureau of Justice Statistics, United States Department of Justice. Criminal victimization in the United States, 1993. A national crime survey report. Washington, DC: Bureau of Justice Statistics, United States Department of Justice (NCJ-151657), 1996.

24 Centers for Disease Control and Prevention. Youth risk behavior surveillance-United States 1995. Morb Mortal Wkly Rep 1996;45(SS-4):1-85.

\section{Editorial Board Member: brief biography}

\section{TSUNG-HSUEH LU}

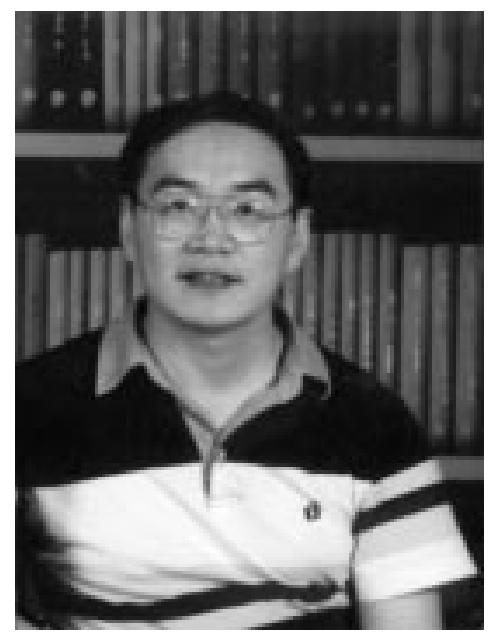

Tsung-Hsueh $\mathrm{Lu}, \mathrm{MD}, \mathrm{MPH}$ is a senior lecturer in the Department of Public Health and an Associate Physician of the Department of Family Medicine of Chung Shan Medical and Dental College Hospital, Taichung, Taiwan.

Dr Lu reported a series of studies on the accuracy of cause-of-death statistics and community diagnosis with a different mortality index. These studies indicated that the problem of injury in Taiwan is very serious, and he then became involved in studying injury epidemiology. After doing some basic mortality studies of injuries in Taiwan, he is now studying the relationship between police traffic casualty data and cause-of-death data. $\mathrm{He}$ is optimistic that the establishment of a linkage data bank will provide more information about the circumstances of fatal traffic injuries. $\mathrm{He}$ is also planning to set up an injury surveillance system in Taiwan.

$\mathrm{He}$ is currently studying for a doctoral degree at the Institute of Health Policy and Management of National Taiwan University. The topic for his dissertation is the social inequality of health and political economy of health with a special interest in injury. 